University of South Florida

DIGITAL COMMONS

Digital Commons @ University of

@ UNIVERSITY OF SOUTH FLORIDA

South Florida

USF Accountability Reports

USF Archives

$1-1-2018$

\title{
2018 Accountability Plan System
}

USF

Follow this and additional works at: https://digitalcommons.usf.edu/usf_accountability_reports

\section{Scholar Commons Citation}

USF, "2018 Accountability Plan System" (2018). USF Accountability Reports. 71.

https://digitalcommons.usf.edu/usf_accountability_reports/71

This Article is brought to you for free and open access by the USF Archives at Digital Commons @ University of South Florida. It has been accepted for inclusion in USF Accountability Reports by an authorized administrator of Digital Commons @ University of South Florida. For more information, please contact digitalcommons@usf.edu. 


\section{8 Accountability Plan}

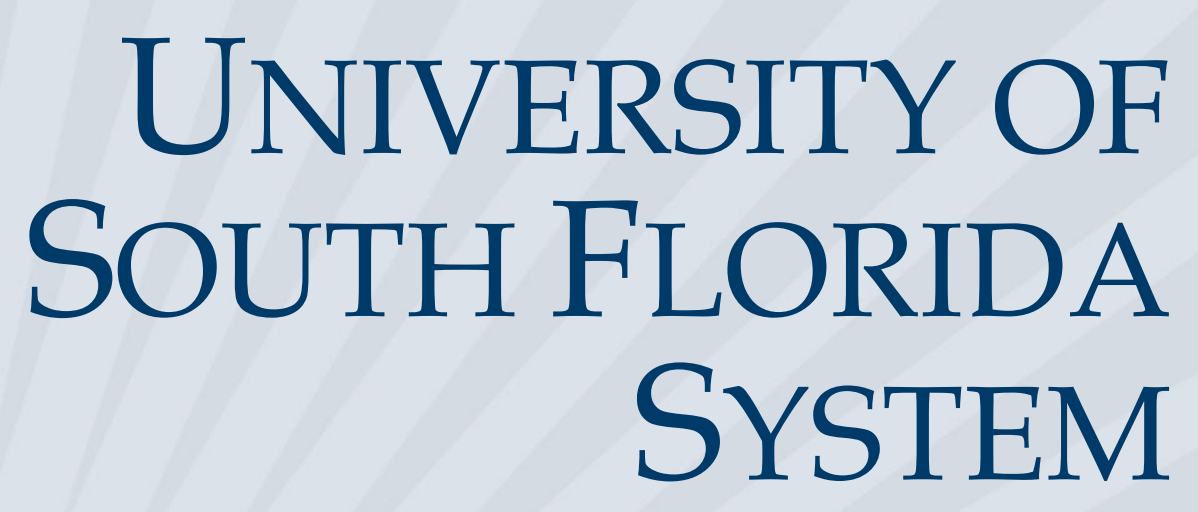

STATE UNIVERSITY SYSTEM of FLORIDA Board of Governors 


\section{INTRODUCTION}

This is a new report that combines the previous Annual Accountability Report and University Work Plans into one new document that is more closely aligned with the Board of Governors' 2025 System Strategic Plan.

This revised document will enhance the System's commitment to accountability and strategic planning by enabling comparisons between past goals and actual data to better assess performance. This change will help foster greater coordination between institutional administrators, University Boards of Trustees and the Board of Governors.

Once an Accountability Plan is approved by each institution's respective Boards of Trustees, the Board of Governors will review and consider the plan for potential acceptance of 2016-17 components. Longer-term components will inform future agendas of the Board's Strategic Planning Committee. The Board's acceptance of a work plan does not constitute approval of any particular component, nor does it supersede any necessary approval processes that may be required for each component. 


\section{TABLE OF CONTENTS}

\section{STRATEGY}

a. Mission \& Vision Statements, p. 3

b. Statement of Strategy, p. 3

c. Strengths and Opportunities, p. 4

d. Key Initiatives \& Investments, p. 5

e. Key Achievements for Last Year, p. 8

2. PERFORMANCE BASED FUNDING METRICS, p. 10-11

3. PREEMINENT RESEARCH UNIVERSITY METRICS, p. 12-14

\section{KEY PERFORMANCE INDICATORS}

a. Teaching \& Learning, p. 15-16

b. Scholarship, Research and Innovation, p. 17-18

c. Institution Specific Goals, p. 19

5. ENROLLMENT PLANNING, p. 20-21

6. ACADEMIC PROGRAM COORDINATION, p. 22-23

7. DEFINITIONS, p.24-29 


\section{MISSION STATEMENT (What is your purpose?)}

The University of South Florida System, which includes USF Tampa, USF St. Petersburg, and USF Sarasota-Manatee, catalyzes and coordinates initiatives at and among its interdependent institutions to prepare students for successful $21^{\text {st }}$ century careers; advances research, scholarship, and creative endeavors to improve the quality of life; and engages its communities for mutual benefit.

\section{VISION STATEMENT (What do you aspire to?)}

The University of South Florida System will empower and connect its institutions into a distinctive system that is nationally and globally recognized for innovation in teaching and research; for attracting outstanding and diverse scholars, staff, and students; and for transforming the communities in which we operate as well as those where our graduates apply their skills.

\section{STATEMENT OF STRATEGY (How will you get there?)}

Given your mission, vision, strengths and available resources, provide a brief description of your market and your strategy for addressing and leading it.

The primary focus of the University of South Florida System is to provide access to high-quality education that promotes student success, research and innovation, and partnerships at regional, national, and global levels. Comprised of USF Tampa, USF St. Petersburg, and USF SarasotaManatee, the University of South Florida System is guided by the State University System of Florida Board of Governors' (BOG) Strategic Plan and driven by a commitment to accountability.

The USF System serves its students by offering diverse solutions to education via course mobility as well as technology- and media-rich online delivery that further increases student success through engaged learning and an expanded portfolio of online offerings. Similarly, the FUSE program-an enhanced transfer agreement between the USF System and eight Florida College System partnerspromotes access to education by offering guaranteed admission to the USF System. The USF System also continues to strengthen its career readiness initiatives including the expansion of experiential learning internship opportunities and career fairs. The USF System maintains partnerships with regional, national, and international organizations to deliver quality internships, research, and experiential learning opportunities that prepare students for lifelong success in today's global workforce.

A prime example of the USF System's continued commitment to seeking new and innovative opportunities and to employing a holistic approach to student success and mental health is the use of predictive analytics to successfully identify students in need of timely interventions and support. By reviewing real-time, individual student data, each of the USF campuses can preemptively engage with its students long before impacting their path to graduation. 


\section{STRENGTHS AND OPPORTUNITIES (within 3 years)}

What are your core capabilities, opportunities and challenges for improvement?

The University of South Florida System's greatest strength is the combined force of its three distinctive campuses, each agile and able to adapt quickly to the evolving needs of its students, local communities and the state of Florida.

In March, Governor Rick Scott signed the Florida Excellence in Higher Education Act of 2018, thereby consolidating the three separate accreditations held by USF Tampa, USF St. Petersburg, and USF Sarasota-Manatee under a single unified accreditation. The consolidated accreditation, which does not go into effect until July 1, 2020, will elevate the success of all students and faculty while upholding the distinctiveness of each campus. The world-class faculty across the USF System are engaged in groundbreaking research that aims to address society's most pervasive problems and create a growing economy for the region, the state, and the nation. The success of the USF System's 50,000 students is paramount and under a consolidated system, USF will deliver expanded programs in areas of strategic emphasis, graduate research and doctoral opportunities responding to the local needs and market demands for each of its communities.

During the implementation process, the USF System's highest priority will remain the success of our students by providing a world-class education. 


\section{KEY INITIATIVES \& INVESTMENTS (within 3 years)}

Describe your top three key initiatives for the next three years that will drive improvement in Academic Quality, Operational Efficiency, and Return on Investment.

\section{Continue to enhance student success to maintain momentum as a top performer in the Board of Governors performance-funding model:}

The USF System provides access to high-quality, globally-informed academic programs and experiential learning opportunities in a continued effort to enhance student success. As a national model of student success and mental health, The USF System employs a holistic approach and supplementing the use of predictive analytics with proactive advising to better understand student decisions and to intervene when necessary. Other programs include the system-wide initiative MWell4Success, which ensures the best services are in place to increase the USF System's capacity to meet the needs of its students, and the Persistence Committee, which includes representation from Advising, Financial Aid, Career Services, the Counseling Center, and Housing, and endeavors to address the myriad of issues that could affect a student's success. Home to a suite of applications and programs that support the university's academic and business processes, Archivum provides additional online platform for faculty and staff to complete tasks and processes as efficiently as possible. The combined effort has resulted in a rise in the USF System's graduation and retention rates, as well as elimination of achievement gap by socioeconomic status.

The USF System Green to Gold Grant, in combination with the Federal Pell Grant, covers tuition, fees, and books for students with no expected family contribution. In an effort to further increase access to higher education and reduce student debt, the Textbook Affordability Project (TAP) endeavors, not only to promote awareness of textbook affordability issues, but also to provide solutions to make course materials accessible and affordable. The USF System has also expanded its portfolio of online offerings across the system, thereby creating a workforce prepared for highskill, high-wage jobs with the businesses that drive today's economy. Greater course mobility and technology- and media-rich online delivery has further increased student success through engaged learning. Finally, Handshake - a cutting-edge technology platform designed connect university students with potential employers - enables the USF System to provide its students and alumni with access to internship and employment opportunities across the globe. 
2. Enhance academic program quality that prepares students for high-skilled, high need jobs:

In an effort to recruit and retain world-class, research-productive faculty talent and advance the USF System's profile, new faculty positions are being strategically distributed across campuses. Students are exposed to multi-level research opportunities and a career-readiness model that focuses on providing them with professional experience and clear pathways from curriculum to career. Newly established pathways include the Tampa campus partnership with technology talent developer Revature, USF St. Petersburg's highly competitive Accelerator Lab, and the Bloomberg data labs located on the USF Sarasota-Manatee campus. These initiatives have strengthened the USF System's reputation as a destination university and improved the faculty-to-student ratio over past years.

The USF System continues to place a high priority on undergraduate research - a critical part of the educational experience, and a proven benefit that provides students with a deeper understanding of their discipline, experience in working collaboratively across disciplines, applied knowledge, and critical in-demand skills such as real-world problem solving and communication.

The new USF Health Heart Institute at USF Tampa's Morsani College of Medicine brings together a critical mass of basic science and clinical researchers to address unmet medical needs related to cardiovascular disease. Led by Dr. Sam Wickline, the Heart Institute reinforces USF's mission to create knowledge, discover solutions to global problems, and prepare students to serve the needs of society. USF Tampa's recently established Department of Medical Engineering, led by faculty from both the College of Engineering and the Morsani College of Medicine, further fosters innovative solutions that save lives and improve the quality of healthcare for all.

In a system-wide effort to combine the science-focused background of Biology with the academic and clinical rigor of Nursing, the Suncoast Nursing Accelerated Pathway (SNAP) allows students to complete a B.S in Biology and a B.S. in Nursing in five years. 


\section{Increase Partnerships and maximize efficiencies:}

To better facilitate working relationships with corporate partners, the USF System launched the Office of Corporate Partnerships at the USF Research Park in Tampa. The office will expand on a strong history of partnering with leading employers in the Tampa Bay Region, and will advance the local economy by growing the workforce and promoting collaborative problem solving.

In conjunction with the Office of Corporate Partnerships, USF Sarasota-Manatee's Business and Education Connection Network (BECN) provides a single university point of contact for current and new community partners to establish and maintain relationships with local organizations and businesses and advance mutually-beneficial goals.

USF St. Petersburg has established strategic corporate partnerships that are thoughtfully integrated with degree programs, research efforts and other campus activities. Such partnerships will help generate research funding, create jobs and internship opportunities for students, provide greater access to specialized facilities, and more. For example, the Innovation District, which houses a cluster of higher education, health care, marine research, media and other key institutions in downtown St. Petersburg has members from USF St. Petersburg on the executive board and innovation council for the district and works with partners including Johns Hopkins All Children's Hospital, Poynter Institute and U.S. Geological Survey to boost collaboration and innovation in the area. 


\section{Key Achievements for 2016-17}

\section{STUDENT ACHIEVEMENTS}

1. Kaitlin Lostroscio, a graduate student in USF Tampa's Mechanical Engineering program, was awarded a NASA Space Technology Research Fellowship (NSTRF) to support her research at USF's Center for Assistive, Rehabilitation, and Robotics Technologies (CAART).

2. Shawna Machado, a former homeless student who made national headlines, graduated from USFSM with a master's degree in social work in spring of 2017.

3. Elementary Education major from USFSP Heather Hammerling received the Fanchon Funk Scholar Award from the Florida Association of Teacher Educators.

\section{FACULTY ACHIEVEMENTS}

1. USFSM's Heather Williams, an accounting instructor in the College of Business, was named "Young Professional of the Year" by the Sarasota Chamber of Commerce.

2. USF Tampa ranked 4th worldwide for organizations with the most AAAS Fellows named in 2016 for the third year in a row. As of 2017, USF had a total of 57 AAAS Fellows.

3. USFSP's Dr. Joseph Smoak received a grant of \$1.3 million from the U.S. Department of Agriculture for his research project titled "Organic Carbon Biomass, Burial, and Biogeochemistry in Blue Carbon Ecosystems Along the South Florida Coast: Climate Change and Anthropogenic Influences.”

\section{PROGRAM ACHIEVEMENTS}

1. USFSM opened the College of Science and Mathematics in 2016 to expand its STEM curriculum and support robust partnerships with regional hospitals and research centers.

2. USFSP offers a new Master of Science in Conservation Biology degree program and is the only M.S. thesis-based degree of its kind in the State University System of Florida.

3. The Morsani College of Medicine at USF Tampa brought in its most selective incoming medical student cohort to date, with an average MCAT score of 514, placing it among the top of all medical schools in the country

\section{RESEARCH ACHIEVEMENTS}

1. USF Tampa was awarded a record $\$ 475.2$ million in research contracts and grants in fiscal year 2017. According to the National Institutes of Health, every $\$ 1.00$ in research funding brings in $\$ 2.21$ in local economic growth. In FY2017, USF's \$475.2 million research funding alone supported more than 5,900 jobs and generated over $\$ 1$ billion in local economic growth.

2. USFSP biologist Dr. Heather Judkins took part in a Deep-Pelagic Nekton Dynamics (DEEPEND) Consortium cruise with researchers from institutions around the U.S. that explored the deep ocean layers of the northern Gulf of Mexico and allowed Judkins to explore, analyze and publish on many aspects of cephalopod systematics, biogeography and other collaborative effects. The DEEPEND Consortium was created in response to the Deepwater Horizon oil spill of 2010 to allow researchers to examine deep sea biodiversity.

3. USFSM's Dr. James Unnever was hired by the Dutch government to provide an official report, "Ethnic Crime in the Netherlands," for the Hague Court, Netherlands (summer 2016). 


\section{INSTITUTIONAL ACHIEVEMENTS}

1. The USF System ranks 5th in the nation among public universities and 11th world-wide for granted U.S. patents among all universities according to the Intellectual Property Owners Association/NAI.

2. USFSM opened the David Kotok and Cumberland Advisors Bloomberg Lab featuring 10 terminals that provide students with access to Bloomberg Professional Services, a powerful resource with realtime data and news from markets around the world.

3 The 68,000-square-foot, state-of-the-art Lynn Pippenger Hall at USFSP opened as the new home of the Kate Tiedemann College of Business in January, 2017. The Hall is named after philanthropist Lynn Pippenger, who gave a \$5 million gift to the institution in 2016.

4 USF Tampa reached \$505.9 Million in total research expenditures in fiscal year 2016 according to the National Science Foundation HERD Survey. 


\section{PERFORMANCE BASED FUNDING METRICS}

1. Percent of Bachelor's Graduates Enrolled or Employed (\$25,000+)

\begin{tabular}{cccccccccc} 
& $2011-12$ & $2012-13$ & $2013-14$ & $2014-15$ & $2015-16$ & $2016-17$ & $2017-18$ & $2018-19$ & $2019-20$ \\
\hline ACTUAL &. & 65.3 & 67.2 & 69.6 & 70.0 &. &. &. &. \\
APPROVED GOALS &. &. &. & 66.8 & 70.5 & 73.0 & 75.0 & 76.0 &. \\
PROPOSED GOALS &. &. &. &. &. & 70.5 & 71.5 & 72.5 & 73.0
\end{tabular}

2. Median Wages of Bachelor's Graduates Employed Full-time

\begin{tabular}{cccccccccc} 
& $2011-12$ & $2012-13$ & $2013-14$ & $2014-15$ & $2015-16$ & $2016-17$ & $2017-18$ & $2018-19$ & $2019-20$ \\
\hline ACTUAL &. & 35,200 & 36,700 & 38,000 & 37,300 &. &. &. &. \\
APPROVED GOALS &. &. &. & 36,300 & 38,600 & 39,100 & 39,600 & 40,400 &. \\
PROPOSED GOALS &. &. &. &. &. & 39,100 & 39,600 & 40,100 & 40,700
\end{tabular}

3. Average Cost to the Student [Net Tuition \& Fees per 120 Credit Hours for Resident Undergraduates]

\begin{tabular}{cccccccccc} 
& $2012-13$ & $2013-14$ & $2014-15$ & $2015-16$ & $\mathbf{2 0 1 6 - 1 7}$ & $\mathbf{2 0 1 7 - 1 8}$ & $\mathbf{2 0 1 8 - 1 9}$ & $\mathbf{2 0 1 9 - 2 0}$ & $\mathbf{2 0 2 0 - 2 1}$ \\
\hline ACTUAL &. & 14,490 & 13,540 & 13,280 & 12,960 &. &. &. & \\
APPROVED GOALS &. &. &. &. & 13,000 & 12,900 & 12,800 & 12,700 &. \\
PROPOSED GOALS &. &. &. &. &. & 12,900 & 12,800 & 12,700 & 12,700
\end{tabular}

\section{FTIC Four-Year Graduation Rate}

\begin{tabular}{lccccccccc} 
& $\mathbf{2 0 0 9 - 1 3}$ & $\mathbf{2 0 1 0 - 1 4}$ & $\mathbf{2 0 1 1 - 1 5}$ & $\mathbf{2 0 1 2 - 1 6}$ & $\mathbf{2 0 1 3 - 1 7}$ & $\mathbf{2 0 1 4 - 1 8}$ & $\mathbf{2 0 1 5 - 1 9}$ & $\mathbf{2 0 1 6 - 2 0}$ & $\mathbf{2 0 1 7 - 2 1}$ \\
\hline $\begin{array}{l}\text { ACTUAL Graduated } \\
\text { Within USF System }\end{array}$ & 41.9 & 43.2 & 48.8 & 52.0 & 57.3 &. &. &. &. \\
APPROVED GOALS &. &. &. & 50.4 & 53.0 & 57.0 & 59.0 & 63.0 &. \\
PROPOSED GOALS &. &. &. &. &. & 57.6 & 59.2 & 61.8 & 64.1
\end{tabular}

5. Academic Progress Rate [Second Year Retention Rate with At Least a 2.0 GPA]

\begin{tabular}{cccccccccc} 
& $2012-13$ & $2013-14$ & $2014-15$ & $2015-16$ & $\mathbf{2 0 1 6 - 1 7}$ & $\mathbf{2 0 1 7 - 1 8}$ & $\mathbf{2 0 1 8 - 1 9}$ & $\mathbf{2 0 1 9 - 2 0}$ & $\mathbf{2 0 2 0 - 2 1}$ \\
\hline $\begin{array}{r}\text { ACTUAL Retained } \\
\text { Within USF System }\end{array}$ & 84.5 & 85.3 & 85.1 & 86.1 & 85.9 &. &. &. &. \\
APPROVED GOALS &. &. &. & 85.6 & 87.5 & 89.0 & 89.5 & 90.0 &. \\
PROPOSED GOALS &. &. &. &. &. & 87.4 & 89.0 & 89.6 & 90.5
\end{tabular}




\section{PERFORMANCE BASED FUNDING METRICS (CONTINUED)}

6. Percentage of Bachelor's Degrees Awarded within Programs of Strategic Emphasis

\begin{tabular}{|c|c|c|c|c|c|c|c|c|c|}
\hline & 2012-13 & 2013-14 & 2014-15 & 2015-16 & 2016-17 & 2017-18 & 2018-19 & $2019-20$ & 2020-21 \\
\hline ACTUAL & 49.5 & 51.0 & 54.6 & 59.0 & 61.4 & . & & & \\
\hline APPROVED GOALS & . & . & . & 54.8 & 59.2 & 59.5 & 60.6 & 61.0 & ${ }^{\circ}$ \\
\hline PROPOSED GOALS & . & . & . & & . & 61.7 & 62.3 & 62.9 & 63.5 \\
\hline 7. University & $\begin{array}{c}\text { ccess I } \\
\text { FALL } \\
2012 \\
\end{array}$ & $\begin{array}{c}\text { te [Perce } \\
\text { FALL } \\
2013 \\
\end{array}$ & $\begin{array}{c}\text { of Underg } \\
\text { FALL } \\
2014 \\
\end{array}$ & $\begin{array}{l}\text { duates w } \\
\text { FALL } \\
\mathbf{2 0 1 5} \\
\end{array}$ & $\begin{array}{c}\text { a Pell gra } \\
\text { FALL } \\
2016 \\
\end{array}$ & $\begin{array}{l}\text { FALL } \\
2017\end{array}$ & $\begin{array}{l}\text { FALL } \\
2018 \\
\end{array}$ & $\begin{array}{l}\text { FALL } \\
2019 \\
\end{array}$ & $\begin{array}{l}\text { FALL } \\
2020 \\
\end{array}$ \\
\hline ACTUAL & 42.0 & 42.1 & 43.0 & 41.2 & 40.0 & . & . & . & 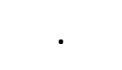 \\
\hline APPROVED GOALS & . & . & . & 40.0 & 41.0 & 41.0 & 41.0 & 41.0 & • \\
\hline PROPOSED GOALS & . & . & 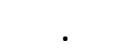 & & . & 40.5 & 40.5 & 40.5 & 40.6 \\
\hline
\end{tabular}

8. Percentage of Graduate Degrees Awarded within Programs of Strategic Emphasis

\begin{tabular}{cccccccccc} 
& $2012-13$ & $2013-14$ & $2014-15$ & $2015-16$ & $2016-17$ & $2017-18$ & $2018-19$ & $2019-20$ & $2020-21$ \\
\hline ACTUAL & 69.1 & 69.0 & 72.7 & 74.6 & 74.8 &. &. &. &. \\
APPROVED GOALS &. &. &. & 74.0 & 74.1 & 74.2 & 74.2 & 74.3 &. \\
PROPOSED GOALS &. &. &. &. &. & 75.2 & 75.3 & 75.3 & 75.8
\end{tabular}

9. BOG Choice: Percent of Baccalaureate Degrees Awarded Without Excess Hours

\begin{tabular}{cccccccccc} 
& $2012-13$ & $2013-14$ & $2014-15$ & $2015-16$ & $2016-17$ & $2017-18$ & $2018-19$ & $2019-20$ & $2020-21$ \\
\hline ACTUAL & 58.2 & 63.9 & 65.8 & 75.6 & 78.3 &. &. &. &. \\
APPROVED GOALS &. &. &. & 68.1 & 77.5 & 78.1 & 79.1 & 80.1 &. \\
PROPOSED GOALS &. &. &. &. &. & 79.0 & 79.6 & 80.2 & 80.3
\end{tabular}

10. BOT Choice: Postdoctoral Appointees

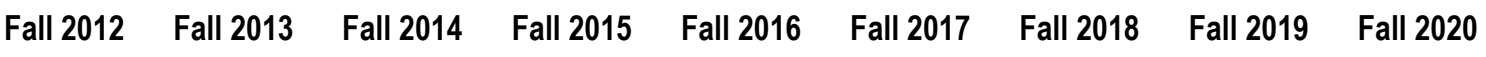

\begin{tabular}{cccccccccc}
\hline ACTUAL & 289 & 321 & 300 & 282 & 272 &. &. &. &. \\
APPROVED GOALS &. &. &. &. & 272 & 267 & 267 &. &. \\
PROPOSED GOALS &. &. &. &. &. & 267 & 267 & 267 & 267
\end{tabular}




\section{PREEMINENT RESEARCH UNIVERSITY FUNDING METRICS (for Tampa Only)}

\section{1a. Average GPA}

\begin{tabular}{cccccccccc} 
& Fall 2013 & Fall 2014 & Fall 2015 & Fall 2016 & Fall 2017 & Fall 2018 & Fall 2019 & Fall 2020 & Fall 2021 \\
\hline ACTUAL & 4.0 & 4.0 & 4.1 & 4.1 & 4.1 &. &. &. &. \\
APPROVED GOALS &. &. &. & 4.0 & 4.1 & 4.1 & 4.1 & 4.1 &. \\
PROPOSED GOALS &. &. &. &. &. & 4.1 & 4.1 & 4.1 & 4.1
\end{tabular}

1b. Average SAT Score

\begin{tabular}{cccccccccc} 
& Fall 2013 & Fall 2014 & Fall 2015 & Fall 2016 & Fall 2017 & Fall 2018 & Fall 2019 & Fall 2020 & Fall 2021 \\
\hline ACTUAL & 1200 & 1197 & 1223 & 1226 & 1280 &. &. &. &. \\
APPROVED GOALS &. &. &. & 1220 & 1280 & 1282 & 1285 & 1290 &. \\
PROPOSED GOALS &. &. &. &. &. & 1282 & 1285 & 1290 & 1290
\end{tabular}

Note* SAT scores reflect rescaling to new SAT standards (approved goals were based upon old standard).

\section{Public University National Ranking [Top50 rankings based on BOG's official list of publications]}

\begin{tabular}{cccccccccc} 
& 2014 & 2015 & 2016 & 2017 & 2018 & 2019 & 2020 & 2021 & 2022 \\
\hline ACTUAL & 1 & 3 & 4 & 4 & 4 &. &. &. &. \\
APPROVED GOALS &. &. &. & 3 & 5 & 5 & 5 & 5 &. \\
PROPOSED GOALS &. &. &. &. &. & 5 & 5 & 5 & 5
\end{tabular}

\section{Freshman Retention Rate [Full-time students as reported to IPEDS]}

\begin{tabular}{cccccccccc} 
& $2012-13$ & $2013-14$ & $2014-15$ & $2015-16$ & $\mathbf{2 0 1 6 - 1 7}$ & $\mathbf{2 0 1 7 - 1 8}$ & $\mathbf{2 0 1 8 - 1 9}$ & $\mathbf{2 0 1 9 - 2 0}$ & $\mathbf{2 0 2 0 - 2 1}$ \\
\hline ACTUAL & 89 & 89 & 88 & 90 & 90 &. &. &. &. \\
APPROVED GOALS &. &. &. & 90 & 91 & 92 & 93 & 93 &. \\
PROPOSED GOALS &. &. &. &. &. & 91 & 91 & 92 & 92
\end{tabular}

\section{Six-year Graduation Rate [Full-time students as reported to IPEDS]}

\begin{tabular}{cccccccccc} 
& $2007-13$ & $2008-14$ & $2009-15$ & $2010-16$ & $2011-17$ & $2012-18$ & $2013-19$ & $2014-20$ & $2015-21$ \\
\hline ACTUAL & 63 & 67 & 68 & 67 & 71 &. &. &. &. \\
APPROVED GOALS &. &. &. & 66 & 71 & 73 & 75 & 77 &. \\
PROPOSED GOALS &. &. &. &. &. & 73 & 75 & 77 & 78
\end{tabular}




\section{PREEMINENT RESEARCH UNIVERSITY FUNDING METRICS (for Tampa Only)}

\section{National Academy Memberships}

\begin{tabular}{cccccccccc} 
& 2014 & 2015 & $\mathbf{2 0 1 6}$ & $\mathbf{2 0 1 7}$ & $\mathbf{2 0 1 8}$ & $\mathbf{2 0 1 9}$ & $\mathbf{2 0 2 0}$ & $\mathbf{2 0 2 1}$ & $\mathbf{2 0 2 2}$ \\
\hline ACTUAL & 6 & 9 & 8 & 11 & 13 &. &. &. &. \\
APPROVED GOALS &. &. &. & 9 & 10 & 10 & 10 & 10 &. \\
PROPOSED GOALS &. &. &. &. &. & 13 & 13 & 13 & 13
\end{tabular}

6. Science \& Engineering Research Expenditures (\$M)

\begin{tabular}{cccccccccc} 
& $2012-13$ & $2013-14$ & $2014-15$ & $2015-16$ & $2016-17$ & $2017-18$ & $2018-19$ & $2019-20$ & $2020-21$ \\
\hline ACTUAL & $\$ 411$ & $\$ 438$ & $\$ 420$ & $\$ 448$ & $\$ 502$ &. &. &. &. \\
APPROVED GOALS &. &. &. & $\$ 421$ & $\$ 427$ & $\$ 434$ & $\$ 440$ & $\$ 447$ &. \\
PROPOSED GOALS &. &. &. &. &. & $\$ 503$ & $\$ 504$ & $\$ 505$ & $\$ 506$
\end{tabular}

7. Non-Medical Science \& Engineering Research Expenditures (\$M)

\begin{tabular}{cccccccccc} 
& $2012-13$ & $2013-14$ & $2014-15$ & $2015-16$ & $2016-17$ & $2017-18$ & $2018-19$ & $2019-20$ & $2020-21$ \\
\hline ACTUAL & $\$ 193$ & $\$ 239$ & $\$ 229$ & $\$ 229$ & $\$ 288$ &. &. &. &. \\
APPROVED GOALS &. &. &. & $\$ 230$ & $\$ 233$ & $\$ 237$ & $\$ 241$ & $\$ 245$ &. \\
PROPOSED GOALS &. &. &. &. &. & $\$ 289$ & $\$ 290$ & $\$ 291$ & $\$ 292$
\end{tabular}

8. Number of Broad Disciplines Ranked in Top 100 for Research Expenditures

\begin{tabular}{cccccccccc} 
& $2011-12$ & $2012-13$ & $2013-14$ & $2014-15$ & $2015-16$ & $2016-17$ & $2017-18$ & $2018-19$ & $2019-20$ \\
\hline ACTUAL & 5 of 8 & 7 of 8 & 7 of 8 & 7 of 8 & 7 of 8 &. &. &. &. \\
APPROVED GOALS &. &. &. & 7 of 8 & 8 of 8 & 8 of 8 & 8 of 8 & 8 of 8 &. \\
PROPOSED GOALS &. &. &. &. &. & 8 of 8 & 8 of 8 & 8 of 8 & 8 of 8
\end{tabular}




\section{PREEMINENT RESEARCH UNIVERSITY FUNDING METRICS (for Tampa Only)}

9. Utility Patents Awarded [over three calendar years]

\begin{tabular}{cccccccccc} 
& $\mathbf{2 0 1 1 - 1 3}$ & $\mathbf{2 0 1 2 - 1 4}$ & $\mathbf{2 0 1 3 - 1 5}$ & $\mathbf{2 0 1 4 - 1 6}$ & $\mathbf{2 0 1 5 - 1 7}$ & $\mathbf{2 0 1 6 - 1 8}$ & $\mathbf{2 0 1 7 - 1 9}$ & $\mathbf{2 0 1 8 - 2 0}$ & $\mathbf{2 0 1 9 - 2 1}$ \\
\hline ACTUAL & 270 & 291 & 297 & 314 & 324 &. &. &. &. \\
APPROVED GOALS &. &. &. & 291 & 273 & 276 & 279 & 282 &. \\
PROPOSED GOALS &. &. &. &. &. & 325 & 325 & 325 & 325
\end{tabular}

10. Doctoral Degrees Awarded Annually

\begin{tabular}{cccccccccc} 
& $2012-13$ & $2013-14$ & $2014-15$ & $2015-16$ & $2016-17$ & $2017-18$ & $2018-19$ & $2019-20$ & $2020-21$ \\
\hline ACTUAL & 448 & 546 & 601 & 704 & 721 &. &. &. &. \\
APPROVED GOALS &. &. &. & 645 & 650 & 655 & 660 & 665 &. \\
PROPOSED GOALS &. &. &. &. &. & 725 & 730 & 735 & 740
\end{tabular}

11. Number of Post-Doctoral Appointees [note: statute requires a source with time lag]

\begin{tabular}{ccccc|c|cccc} 
& Fall 2010 & Fall 2011 & Fall 2012 & Fall 2013 & $\begin{array}{c}\text { Fall 2014 } \\
\text { Official }\end{array}$ & Fall 2015 & Fall 2016 & Fall 2017 & Fall 2018 \\
\hline ACTUAL & 293 & 304 & 289 & 321 & 300 &. &. &. &. \\
APPROVED GOALS &. &. &. & 321 & 300 & 277 & 267 & 260 &. \\
PROPOSED GOALS &. &. &. &. &. & 277 & 267 & 260 & 260
\end{tabular}

\section{Endowment Size (\$Millions)}

\begin{tabular}{cccccccccc} 
& $2012-13$ & $2013-14$ & $2014-15$ & $2015-16$ & $2016-17$ & $2017-18$ & $2018-19$ & $2019-20$ & $2020-21$ \\
\hline ACTUAL & 364 & 417 & 417 & 395 & 442 &. &. &. &. \\
APPROVED GOALS &. &. &. & 395 & 412 & 432 & 448 & 472 &. \\
PROPOSED GOALS &. &. &. &. &. & 450 & 465 & 485 & 500
\end{tabular}




\section{KEY PERFORMANCE INDICATORS}

Teaching \& Learning Metrics (from the 2025 System Strategic Plan that are not included in the PBF section)

Public University National Ranking [Number of Top50 Rankings based on BOG's official list of publications]

\begin{tabular}{cccccccccc} 
& 2014 & 2015 & 2016 & 2017 & 2018 & 2019 & 2020 & 2021 & 2022 \\
\hline ACTUAL & 1 & 3 & 4 & 4 & 4 &. &. &. &. \\
APPROVED GOALS &. &. &. & 5 & 5 & 5 & 5 & 5 &. \\
PROPOSED GOALS &. &. &. &. &. & 5 & 5 & 5 & 5
\end{tabular}

Freshmen in Top 10\% of High School Class

Fall 2013 Fall 2014 Fall 2015 Fall $2016 \quad$ Fall $2017 \quad$ Fall 2018 Fall 2019 Fall 2020 Fall 2021

\begin{tabular}{|c|c|c|c|c|c|c|c|c|c|}
\hline ACTUAL & 36.3 & 28.0 & 30.0 & 33.0 & 33.8 & . & . & . & . \\
\hline APPROVED GOALS & . & . & . & 30.4 & 33.0 & 33.0 & 33.0 & 33.0 & . \\
\hline PROPOSED GOALS & . & . & . & . & . & 34.0 & 34.5 & 35.0 & 35.5 \\
\hline \multicolumn{10}{|c|}{ Professional Licensure \& Certification Exam First-time Pass Rates } \\
\hline CALENDAR YEAR & 2013 & 2014 & 2015 & 2016 & 2017 & $\begin{array}{c}2018 \\
\text { GOALS }\end{array}$ & $\begin{array}{c}2019 \\
\text { GOALS }\end{array}$ & $\begin{array}{c}2020 \\
\text { GOALS }\end{array}$ & $\begin{array}{c}2021 \\
\text { GOALS }\end{array}$ \\
\hline Nursing & $91 \%$ & $86 \%$ & $90 \%$ & $94 \%$ & $93 \%$ & $100 \%$ & $100 \%$ & $100 \%$ & $100 \%$ \\
\hline US Average & $85 \%$ & $85 \%$ & $87 \%$ & $85 \%$ & $87 \%$ & . & . & . & . \\
\hline Medicine (2Y) & $96 \%$ & $95 \%$ & $96 \%$ & $94 \%$ & $92 \%$ & $100 \%$ & $100 \%$ & $100 \%$ & $100 \%$ \\
\hline US Average & $97 \%$ & $96 \%$ & $95 \%$ & $96 \%$ & $96 \%$ & . & . & . & . \\
\hline Pharmacy & . & . & $94 \%$ & $91 \%$ & $86 \%$ & $100 \%$ & $100 \%$ & $100 \%$ & $100 \%$ \\
\hline US Average & $95 \%$ & $95 \%$ & $93 \%$ & $86 \%$ & $88 \%$ & . & . & . & e \\
\hline CROSS-YEAR & 2012-13 & 2013-14 & 2014-15 & 2015-16 & 2016-17 & $\begin{array}{c}2018 \\
\text { GOALS }\end{array}$ & $\begin{array}{c}2019 \\
\text { GOALS }\end{array}$ & $\begin{array}{c}2020 \\
\text { GOALS }\end{array}$ & $\begin{array}{c}2021 \\
\text { GOALS }\end{array}$ \\
\hline Medicine (CK) & $100 \%$ & $98 \%$ & $97 \%$ & $99 \%$ & $95 \%$ & $100 \%$ & $100 \%$ & $100 \%$ & $100 \%$ \\
\hline US Average & $98 \%$ & $97 \%$ & $95 \%$ & $96 \%$ & $96 \%$ & . & . & . & . \\
\hline Medicine (CS) & $99 \%$ & $91 \%$ & $96 \%$ & $97 \%$ & $96 \%$ & $100 \%$ & $100 \%$ & $100 \%$ & $100 \%$ \\
\hline US Average & $98 \%$ & $96 \%$ & $96 \%$ & $97 \%$ & $96 \%$ & . & . & . & . \\
\hline MULTI-YEAR & 2011-13 & 2012-14 & 2013-15 & 2014-16 & 2015-17 & $\begin{array}{c}2018 \\
\text { GOALS }\end{array}$ & $\begin{array}{c}2019 \\
\text { GOALS }\end{array}$ & $\begin{array}{c}2020 \\
\text { GOALS }\end{array}$ & $\begin{array}{c}2021 \\
\text { GOALS }\end{array}$ \\
\hline Physical Therapy & $94 \%$ & $97 \%$ & $95 \%$ & $94 \%$ & $95 \%$ & $100 \%$ & $100 \%$ & $100 \%$ & $100 \%$ \\
\hline US Average & $89 \%$ & $90 \%$ & $91 \%$ & $92 \%$ & $92 \%$ & . & . & . & . \\
\hline \multicolumn{10}{|c|}{ Exam Scores Relative to Benchmarks } \\
\hline Above or Tied & 4 & 3 & 6 & 5 & 3 & 6 & 6 & 6 & 6 \\
\hline Below & 1 & 2 & 0 & 1 & 3 & 0 & 0 & 0 & 0 \\
\hline
\end{tabular}

Note: An asterisk (*) indicates the pass rate is preliminary. 


\section{KEY PERFORMANCE INDICATORS (CONTINUED)}

\section{Teaching \& Learning Metrics}

Time to Degree for FTICs in $120 \mathrm{hr}$ programs

2012-13 2013-14 2014-15 2015-1

2016-17 2017-18

2018-19

2019-20 2020-21

ACTUAL

5.1

4.7

4.5

4.3

4.5

APPROVED GOALS

PROPOSED GOALS
4.5
4.3

4.2
4.2

4.2

4.2

4.2

Six-Year FTIC Graduation Rates [includes full- \& part-time students]

\begin{tabular}{lccccccccc} 
& $2007-13$ & $2008-14$ & $2009-15$ & $2010-16$ & $\mathbf{2 0 1 1 - 1 7}$ & $\mathbf{2 0 1 2 - 1 8}$ & $\mathbf{2 0 1 3 - 1 9}$ & $\mathbf{2 0 1 4 - 2 0}$ & $\mathbf{2 0 1 5 - 2 1}$ \\
\hline $\begin{array}{l}\text { ACTUAL Graduated } \\
\text { Within USF System }\end{array}$ & 63.2 & 66.1 & 67.8 & 66.3 & 68.8 &. &. &. &. \\
APPROVED GOALS &. &. &. & 66.7 & 70.0 & 71.0 & 72.0 & 74.0 &. \\
PROPOSED GOALS &. &. &. &. &. & 69.0 & 71.0 & 74.0 & 75.0
\end{tabular}

Bachelor's Degrees Awarded [First Majors Only]

\begin{tabular}{cccccccccc} 
& $2012-13$ & $2013-14$ & $2014-15$ & $2015-16$ & $\mathbf{2 0 1 6 - 1 7}$ & $\mathbf{2 0 1 7 - 1 8}$ & $\mathbf{2 0 1 8 - 1 9}$ & $\mathbf{2 0 1 9 - 2 0}$ & $\mathbf{2 0 2 0 - 2 1}$ \\
\hline ACTUAL & 8,999 & 9,390 & 9,290 & 9,222 & 9,504 &. &. &. & \\
APPROVED GOALS &. &. &. & 9,081 & 9,255 & 9,285 & 9,416 & 9,557 &. \\
PROPOSED GOALS &. &. &. &. &. & 9,513 & 9,595 & 9,632 & 9,723
\end{tabular}

Graduate Degrees Awarded [First Majors Only]

\begin{tabular}{cccccccccc} 
& $2012-13$ & $2013-14$ & $2014-15$ & $2015-16$ & $2016-17$ & $2017-18$ & $2018-19$ & $2019-20$ & $2020-21$ \\
\hline ACTUAL & 3,209 & 3,401 & 3,773 & 3,918 & 4,208 &. &. &. & \\
APPROVED GOALS &. &. &. & 3,877 & 3,973 & 4,056 & 4,113 & 4,172 &. \\
PROPOSED GOALS &. &. &. &. &. & 4,233 & 4,246 & 4,259 & 4,279
\end{tabular}

Bachelor's Degrees Awarded to African-American \& Hispanic Students

\begin{tabular}{cccccccccc} 
& $2012-13$ & $2013-14$ & $2014-15$ & $2015-16$ & $2016-17$ & $\mathbf{2 0 1 7 - 1 8}$ & $\mathbf{2 0 1 8 - 1 9}$ & $\mathbf{2 0 1 9 - 2 0}$ & $2020-21$ \\
\hline ACTUAL & 27.6 & 28.7 & 30.4 & 31.3 & 31.5 &. &. &. &. \\
APPROVED GOALS &. &. &. & 29.6 & 31.3 & 31.6 & 31.8 & 31.8 &. \\
PROPOSED GOALS &. &. &. &. &. & 32.0 & 32.0 & 32.0 & 32.0
\end{tabular}




\section{KEY PERFORMANCE INDICATORS (CONTINUED)}

\section{Teaching \& Learning Metrics}

Percentage of Adult (Aged 25+) Undergraduates Enrolled

\begin{tabular}{cccccccccc} 
& Fall 2013 & Fall 2014 & Fall 2015 & Fall 2016 & Fall 2017 & Fall 2018 & Fall 2019 & Fall 2020 & Fall 2021 \\
\hline ACTUAL & 24 & 24 & 23 & 22 & 22 &. &. &. &. \\
APPROVED GOALS &. &. &. & 23 & 22 & 22 & 20 & 21 &. \\
PROPOSED GOALS &. &. &. &. &. & 20 & 21 & 21 & 21
\end{tabular}

Percent of Undergraduate FTE in Online Courses

\begin{tabular}{cccccccccc} 
& $2012-13$ & $2013-14$ & $2014-15$ & $2015-16$ & $2016-17$ & $2017-18$ & $2018-19$ & $2019-20$ & $2020-21$ \\
\hline ACTUAL & 23 & 23 & 26 & 28 & 29 &. &. &. &. \\
APPROVED GOALS &. &. &. & 27.5 & 28 & 29 & 30 & 30 & $\cdot$ \\
PROPOSED GOALS &. &. &. &. &. & 30 & 31 & 32 & 33
\end{tabular}

Percent of Bachelor's Degrees in STEM \& Health

\begin{tabular}{cccccccccc} 
& $2012-13$ & $2013-14$ & $2014-15$ & $2015-16$ & $2016-17$ & $2017-18$ & $2018-19$ & $2019-20$ & $2020-21$ \\
\hline ACTUAL & 32 & 34 & 38 & 42 & 44 &. &. &. &. \\
APPROVED GOALS &. &. &. & 41 & 42 & 43 & 44 & 44 &. \\
PROPOSED GOALS &. &. &. &. &. & 44 & 45 & 45 & 45
\end{tabular}

Percent of Graduate Degrees in STEM \& Health

\begin{tabular}{cccccccccc} 
& $2012-13$ & $2013-14$ & $2014-15$ & $2015-16$ & $2016-17$ & $2017-18$ & $2018-19$ & $2019-20$ & $2020-21$ \\
\hline ACTUAL & 50 & 52 & 57 & 60 & 63 &. &. &. &. \\
APPROVED GOALS &. &. &. & 60 & 61 & 62 & 63 & 63 &. \\
PROPOSED GOALS &. &. &. &. &. & 63 & 63 & 64 & 64
\end{tabular}

Scholarship, Research and Innovation Metrics

National Academy Memberships

\begin{tabular}{cccccccccc} 
& 2014 & 2015 & 2016 & 2017 & 2018 & 2019 & $\mathbf{2 0 2 0}$ & $\mathbf{2 0 2 1}$ & 2022 \\
\hline ACTUAL & 6 & 7 & 8 & 11 & 13 &. &. &. &. \\
APPROVED GOALS &. &. &. & 9 & 10 & 10 & 10 & 10 &. \\
PROPOSED GOALS &. &. &. &. &. & 13 & 13 & 13 & 13
\end{tabular}

Faculty Awards

\begin{tabular}{cccccccccc} 
& Fall 2011 & Fall 2012 & Fall 2013 & Fall 2014 & Fall 2015 & Fall 2016 & Fall 2017 & Fall 2018 & Fall 2019 \\
\hline ACTUAL & 10 & 5 & 8 & 8 & 13 &. &. &. &. \\
APPROVED GOALS &. &. &. & 7 & 8 & 9 & 10 & 11 &. \\
PROPOSED GOALS &. &. &. &. &. & 9 & 10 & 11 & 11
\end{tabular}




\section{KEY PERFORMANCE INDICATORS (CONTINUED)}

\section{Scholarship, Research and Innovation Metrics}

Total Research Expenditures (\$M)

\begin{tabular}{cccccccccc} 
& $2012-13$ & $2013-14$ & $2014-15$ & $2015-16$ & $2016-17$ & $2017-18$ & $2018-19$ & $2019-20$ & $2020-21$ \\
\hline ACTUAL & $\$ 467$ & $\$ 497$ & $\$ 494$ & $\$ 515$ & $\$ 568$ &. &. &. &. \\
APPROVED GOALS &. &. &. & $\$ 495$ & $\$ 510$ & $\$ 525$ & $\$ 541$ & $\$ 557$ &. \\
PROPOSED GOALS &. &. &. &. &. & $\$ 569$ & $\$ 570$ & $\$ 571$ & $\$ 572$
\end{tabular}

Percentage of Research Expenditures Funded from External Sources

\begin{tabular}{|c|c|c|c|c|c|c|c|c|c|}
\hline & 2012-13 & 2013-14 & 2014-15 & 2015-16 & 2016-17 & $2017-18$ & 2018-19 & 2019-20 & 2020-21 \\
\hline ACTUAL & 59 & 60 & 55 & 55 & 57 & . & . & . & . \\
\hline APPROVED GOALS & . & . & . & 56 & 57 & 58 & 59 & 60 & . \\
\hline PROPOSED GOALS & . & . & . & . & . & 58 & 59 & 60 & 60 \\
\hline \multicolumn{10}{|c|}{ Utility Patents Awarded [from the USPTO] } \\
\hline & 2013 & 2014 & 2015 & 2016 & 2017 & 2018 & 2019 & 2020 & 2021 \\
\hline ACTUAL & 98 & 110 & 90 & 114 & 120 & . & . & . & . \\
\hline APPROVED GOALS & . & . & . & . & 69 & 93 & 117 & 72 & . \\
\hline PROPOSED GOALS & . & . & . & . & . & 121 & 122 & 122 & 122 \\
\hline
\end{tabular}

Number of Licenses/Options Executed Annually

\begin{tabular}{cccccccccc} 
& $2012-13$ & $2013-14$ & $2014-15$ & $2015-16$ & $2016-17$ & $2017-18$ & $2018-19$ & $2019-20$ & $\mathbf{2 0 2 0 - 2 1}$ \\
\hline ACTUAL & 75 & 91 & 119 & 133 & 122 &. &. &. &. \\
APPROVED GOALS &. &. & 119 & 120 & 121 & 122 & 123 &. &. \\
PROPOSED GOALS &. &. &. &. &. & 123 & 123 & 123 & 123
\end{tabular}

Number of Start-up Companies Created

\begin{tabular}{cccccccccc} 
& $2012-13$ & $2013-14$ & $2014-15$ & $2015-16$ & $2016-17$ & $2017-18$ & $2018-19$ & $2019-20$ & $2020-21$ \\
\hline ACTUAL & 9 & 11 & 11 & 9 & 10 &. &. &. & \\
APPROVED GOALS &. &. & 8 & 8 & 9 & 10 & 11 &. &. \\
PROPOSED GOALS &. &. &. &. &. & 10 & 11 & 11 & 11
\end{tabular}




\section{KEY PERFORMANCE INDICATORS (CONTINUED)}

Institution Specific Goals

To further distinguish the university's distinctive mission, the university may choose to provide additional metric goals that are based on the university's own strategic plan.

\section{SEE INDIVIDUAL USF SYSTEM MEMBER INSTITUTION ACCOUNTABILITY PLANS}




\section{ENROLLMENT PLANNING}

Headcount Enrollment by Student Type (for all students at all campuses)

\begin{tabular}{|c|c|c|c|c|c|c|c|c|c|}
\hline & $\begin{array}{c}\text { FALL } \\
2013 \\
\text { ACTUAL }\end{array}$ & $\begin{array}{c}\text { FALL } \\
2014 \\
\text { ACTUAL } \\
\end{array}$ & $\begin{array}{c}\text { FALL } \\
2015 \\
\text { ACTUAL } \\
\end{array}$ & $\begin{array}{c}\text { FALL } \\
2016 \\
\text { ACTUAL } \\
\end{array}$ & $\begin{array}{c}\text { FALL } \\
2017 \\
\text { ACTUAL } \\
\end{array}$ & $\begin{array}{l}\text { FALL } \\
2018 \\
\text { PLAN }\end{array}$ & $\begin{array}{l}\text { FALL } \\
2019 \\
\text { PLAN }\end{array}$ & $\begin{array}{l}\text { FALL } \\
2020 \\
\text { PLAN }\end{array}$ & $\begin{array}{l}\text { FALL } \\
2021 \\
\text { PLAN }\end{array}$ \\
\hline \multicolumn{10}{|l|}{ UNDERGRADUATE } \\
\hline FTIC (Regular Admit) & 17,062 & 17,176 & 17,499 & 17,816 & 17,988 & 18,136 & 18,344 & 18,609 & 18,907 \\
\hline FTIC (Profile Admit) & 155 & 202 & 205 & 207 & 201 & 210 & 214 & 216 & 220 \\
\hline FCS AA Transfers & 9,498 & 9,416 & 9,172 & 9,108 & 9,416 & 9,243 & 9,136 & 9,074 & 9,089 \\
\hline Other AA Transfers & 1,640 & 1,565 & 1,495 & 1,429 & 1,511 & 1,523 & 1,546 & 1,569 & 1,596 \\
\hline Post-Baccalaureates & 0 & 1,110 & 1,025 & 998 & 986 & 999 & 1,015 & 1,031 & 1,046 \\
\hline Other Undergraduates & 7,739 & 6,583 & 6,658 & 6,678 & 6,853 & 6,738 & 6,791 & 6,895 & 6,969 \\
\hline Subtotal & 36,012 & 35,808 & 35,990 & 36,373 & 36,955 & 36,850 & 37,044 & 37,395 & 37,826 \\
\hline \multicolumn{10}{|l|}{ GRADUATE } \\
\hline Master's & 6,806 & 6,950 & 7,160 & 7,302 & 7,690 & 7,745 & 7,818 & 7,910 & 8,006 \\
\hline Research Doctoral & 2,294 & 2,226 & 2,229 & 2,333 & 2,443 & 2,453 & 2,463 & 2,473 & 2,482 \\
\hline Professional Doctoral & 1,235 & 1,379 & 1,309 & 1,348 & 1,436 & 1,460 & 1,484 & 1,509 & 1,534 \\
\hline Subtotal & 10,335 & 10,555 & 10,698 & 10,983 & 11,569 & 11,657 & 11,765 & 11,891 & 12,023 \\
\hline \multicolumn{10}{|l|}{ UNCLASSIFIED } \\
\hline H.S. Dual Enrolled & 42 & 14 & 24 & 31 & 33 & 35 & 39 & 43 & 47 \\
\hline Other $^{1}$ & 1,941 & 2,201 & 2,272 & 2,424 & 2,227 & 2,291 & 2,344 & 2,390 & 2,440 \\
\hline Subtotal & 1,983 & 2,215 & 2,296 & 2,455 & 2,260 & 2,326 & 2,383 & 2,433 & 2,487 \\
\hline TOTAL & 48,330 & 48,578 & 48,984 & 49,811 & 50,784 & 50,832 & 51,193 & 51,720 & 52,336 \\
\hline
\end{tabular}

Notes: This table reports the number of students enrolled at the university by student type categories. The student type for undergraduates is based on the Type of Student at Time of Most Recent Admission. The student type for graduates is based on the degree that is sought and the student CIP code. Unclassified refers to a student who has not yet been formally admitted into a degree program but is enrolled. (1) 'Other Unclassified' students include Post-Baccalaureates who are not seeking a degree. 
ENROLLMENT PLANNING (CONTINUED) FTE Enrollment by Residency \& Student Level

\begin{tabular}{|c|c|c|c|c|c|c|c|c|c|c|}
\hline & $\begin{array}{l}2012-13 \\
\text { ACTUAL }\end{array}$ & $\begin{array}{l}\text { 2013-14 } \\
\text { ACTUAL }\end{array}$ & $\begin{array}{l}\text { 2014-15 } \\
\text { ACTUAL }\end{array}$ & $\begin{array}{l}2015-16 \\
\text { ACTUAL }\end{array}$ & $\begin{array}{l}\text { 2016-17 } \\
\text { ACTUAL }\end{array}$ & $\begin{array}{c}2017-18 \\
\text { PLAN }\end{array}$ & $\begin{array}{l}2018-19 \\
\text { PLAN }\end{array}$ & $\begin{array}{l}2019-20 \\
\text { PLAN }\end{array}$ & $\begin{array}{l}2020-21 \\
\text { PLAN }\end{array}$ & $\begin{array}{l}2021-22 \\
\text { PLAN }\end{array}$ \\
\hline \multicolumn{11}{|l|}{ RESIDENT } \\
\hline LOWER & 12,372 & 12,087 & 11,844 & 11,933 & 12,066 & 12,007 & 12,134 & 12,334 & 12,595 & 12,872 \\
\hline UPPER & 19,921 & 19,341 & 18,957 & 18,827 & 18,338 & 18,765 & 18,738 & 18,605 & 18,458 & 18,479 \\
\hline GRAD I & 4,772 & 4,794 & 4,744 & 4,568 & 4,339 & 4,403 & 4,495 & 4,544 & 4,594 & 4,646 \\
\hline GRAD ॥ & 1,291 & 1,266 & 1,279 & 1,199 & 1,219 & 1,267 & 1,329 & 1,339 & 1,353 & 1,366 \\
\hline TOTAL & 38,357 & 37,487 & 36,825 & 36,528 & 35,962 & 36,442 & 36,696 & 36,822 & 37,001 & 37,364 \\
\hline
\end{tabular}

NON-RESIDENT

\begin{tabular}{lrrrrr|rrrrr} 
LOWER & 1,119 & 1,357 & 1,574 & 1,862 & 1,992 & 1,957 & 1,911 & 1,934 & 1,966 & 1,998 \\
UPPER & 870 & 1,058 & 1,304 & 1,596 & 1,819 & 2,007 & 1,727 & 1,707 & 1,693 & 1,692 \\
GRAD I & 1,061 & 1,370 & 1,652 & 1,984 & 2,142 & 2,112 & 2,094 & 2,117 & 2,141 & 2,165 \\
GRAD II & 843 & 853 & 880 & 935 & 1,017 & 1,123 & 1073 & 1,081 & 1,092 & 1,103 \\
TOTAL & $\mathbf{3 , 8 9 3}$ & $\mathbf{4 , 6 3 8}$ & $\mathbf{5 , 4 1 1}$ & $\mathbf{6 , 3 7 7}$ & $\mathbf{6 , 9 7 0}$ & $\mathbf{7 , 1 9 9}$ & $\mathbf{6 , 8 0 5}$ & $\mathbf{6 , 8 3 9}$ & $\mathbf{6 , 8 9 2}$ & $\mathbf{6 , 9 5 7}$
\end{tabular}

TOTAL

\begin{tabular}{lrrrrr|rrrrr} 
LOWER & 13,491 & 13,443 & 13,419 & 13,795 & 14,057 & 13,964 & 14,044 & 14,268 & 14,561 & 14,870 \\
UPPER & 20,790 & 20,400 & 20,262 & 20,423 & 20,158 & 20,772 & 20,465 & 20,311 & 20,151 & 20,171 \\
GRAD I & 5,834 & 6,164 & 6,396 & 6,553 & 6,482 & 6,515 & 6,589 & 6,661 & 6,736 & 6,811 \\
GRAD II & 2,135 & 2,118 & 2,159 & 2,134 & 2,236 & 2,391 & 2,402 & 2,420 & 2,445 & 2,469 \\
TOTAL & $\mathbf{4 2 , 2 5 0}$ & $\mathbf{4 2 , 1 2 5}$ & $\mathbf{4 2 , 2 3 6}$ & $\mathbf{4 2 , 9 0 5}$ & $\mathbf{4 2 , 9 3 2}$ & $\mathbf{4 3 , 6 4 1}$ & $\mathbf{4 3 , 5 0 1}$ & $\mathbf{4 3 , 6 6 1}$ & $\mathbf{4 3 , 8 9 3}$ & $\mathbf{4 4 , 3 2 1}$
\end{tabular}

Note: Full-time Equivalent (FTE) student is a measure of all instructional activity (regardless of fundability) that is based on the number of credit hours that students enroll. FTE is based on the standard national definition, which divides undergraduate credit hours by 30 and graduate credit hours by 24 . Pursuant to section 1013.31, Florida Statutes, Board facilities staff use this data as a key factor in the calculation of facility space needs for university educational plant surveys.

\section{FTE Enrollment by Method of Instruction (for all students at all campuses)}

\begin{tabular}{|c|c|c|c|c|c|c|c|c|c|c|}
\hline & $\begin{array}{l}2012-13 \\
\text { ACTUAL }\end{array}$ & $\begin{array}{l}2013-14 \\
\text { ACTUAL }\end{array}$ & $\begin{array}{l}2014-15 \\
\text { ACTUAL }\end{array}$ & $\begin{array}{l}2015-16 \\
\text { ACTUAL }\end{array}$ & $\begin{array}{l}2016-17 \\
\text { ACTUAL }\end{array}$ & $\begin{array}{c}2017-18 \\
\text { PLAN }\end{array}$ & $\begin{array}{c}2018-19 \\
\text { PLAN }\end{array}$ & $\begin{array}{c}2019-20 \\
\text { PLAN }\end{array}$ & $\begin{array}{c}2020-21 \\
\text { PLAN }\end{array}$ & $\begin{array}{c}2021-22 \\
\text { PLAN }\end{array}$ \\
\hline \multicolumn{11}{|l|}{ UNDERGRADUATE } \\
\hline Distance (80-100\%) & 7,804 & 7,867 & 8,745 & 9,442 & 10,070 & 10,421 & 10,702 & 10,976 & 11,369 & 11,658 \\
\hline Hybrid (50-79\%) & 670 & 580 & 522 & 237 & 183 & 133 & 134 & 137 & 139 & 142 \\
\hline Classroom (0-50\%) & 25,807 & 25,396 & 24,413 & 24,540 & 23,961 & 24,181 & 23,674 & 23,426 & 23,205 & 2,3241 \\
\hline Subtotal & 34,281 & 33,843 & 33,680 & 34,219 & 34,215 & 34,735 & 34,510 & 34,539 & 34,712 & 35,041 \\
\hline \multicolumn{11}{|l|}{ GRADUATE } \\
\hline Distance (80-100\%) & 1,747 & 1,825 & 2,050 & 2,109 & 2,346 & 2,377 & 2,450 & 2,521 & 2,596 & 2,674 \\
\hline Hybrid (50-79\%) & 215 & 234 & 204 & 69 & 123 & 122 & 123 & 124 & 125 & 126 \\
\hline Classroom (0-50\%) & 6,006 & 6,223 & 6,300 & 6,510 & 6,248 & 6,407 & 6,419 & 6,437 & 6,460 & 6,481 \\
\hline Subtotal & 7,968 & 8,282 & 8,554 & 8,688 & 8,718 & 8,906 & 8,991 & 9,081 & 9,181 & 9,280 \\
\hline
\end{tabular}

Note: Full-time Equivalent (FTE) student is a measure of instructional activity (regardless of fundability) that is based on the number of credit hours that students enroll. FTE is based on the standard national definition, which divides undergraduate credit hours by 30 and graduate credit hours by 24 . Distance Learning is a course in which at least 80 percent of the direct instruction of the course is delivered using some form of technology when the student and instructor are separated by time or space, or both (per 1009.24(17), F.S.). Classroom/Traditional, is a course in which less than $50 \%$ of the direct instruction of the course is delivered using some form of technology when the student and instructor are separated by time, space or both. This designation can include activities that do not occur in a classroom (ie, labs, internships, practica, clinicals, labs, etc) - see SUDS data element \#2052. 


\section{ACADEMIC PROGRAM COORDINATION}

\section{New Programs For Consideration by University in AY 2018-19}

The S.U.S. Council of Academic Vice Presidents (CAVP) Academic Program Coordination Work Group will review these programs as part of their on-going coordination efforts. The programs listed below are based on the 2017 Work Plan list for programs under consideration for 2018-20.

\begin{tabular}{|c|c|c|c|c|c|c|}
\hline \multirow[b]{5}{*}{ בזודנדיד } & \multirow{5}{*}{$\begin{array}{c}\text { CIP CODE } \\
\text { 6-digit }\end{array}$} & \multirow{2}{*}{\multicolumn{5}{|c|}{ OFFERED }} \\
\hline & & \multirow{2}{*}{ AREA OF } & & & & \\
\hline & & & UINIVERSITIES & VIA & PROJECTED & PROPOSED \\
\hline & & STRATEGIC & WITH SAME & LEARNING & ENROLLMENT & SUBMISSION \\
\hline & & EMPHASIS & PROGRAM & IN SYSTEM & in 5 th year & TO UBOT \\
\hline \multicolumn{7}{|l|}{ BACHELOR'S PROGRAMS } \\
\hline $\begin{array}{l}\text { Computer and IS Security/Inform } \\
\text { Assurance (USFT) }\end{array}$ & 11.1003 & STEM & None & $80 \%$ & 150 & Fall 2018 \\
\hline Environmental Chemistry (USFSP) & 40.0509 & STEM & None & $10 \%$ & 50 & Spring 2019 \\
\hline $\begin{array}{l}\text { Logistics, Materials \& Supply Chain } \\
\text { Management (USFT) }\end{array}$ & 52.0203 & STEM & $\begin{array}{l}\text { FAMU, FPU, } \\
\text { UNF, UWF }\end{array}$ & $0 \%$ & 150 & Fall 2018 \\
\hline Financial Planning and Services (USFT) & 52.0804 & N/A & UNF & $0 \%$ & 80 & Fall 2018 \\
\hline Management Science (USFSM) & 52.1301 & STEM & UF, FIU & $100 \%$ & 25 & Spring 2019 \\
\hline
\end{tabular}

\section{MASTER'S, SPECIALIST AND OTHER ADVANCED MASTER'S PROGRAMS}

Logistics, Materials \& Supply Chain

Management (USFT)

52.0203 STEM

FAMU

$65 \%$

50

Fall 2018

\section{DOCTORAL PROGRAMS}

Informatics (USFT)

11.0104

STEM

UF

$15 \%$

25

Fall 2018 


\section{New Programs For Consideration by University in 2019-20}

These programs will be used in the 2017-18 Accountability Plan list for programs under consideration for 2019-20.

\begin{tabular}{|c|c|c|c|c|c|c|}
\hline PROGRAM TITLES & $\begin{array}{l}\text { CIP CODE } \\
\text { 6-digit }\end{array}$ & $\begin{array}{c}\text { AREA OF } \\
\text { STRATEGIC } \\
\text { EMPHASIS }\end{array}$ & $\begin{array}{l}\text { OTHER } \\
\text { UNIVERSITIES } \\
\text { WITH SAME } \\
\text { PROGRAM }\end{array}$ & $\begin{array}{c}\text { OFFERED } \\
\text { VIA } \\
\text { DISTANCE } \\
\text { LEARNING } \\
\text { IN SYSTEM }\end{array}$ & $\begin{array}{c}\text { PROJECTED } \\
\text { ENROLLMENT } \\
\text { in 5th year }\end{array}$ & $\begin{array}{l}\text { PROPOSED DATE } \\
\text { OF SUBMISSION } \\
\text { TO UBOT }\end{array}$ \\
\hline \multicolumn{7}{|l|}{ BACHELOR'S PROGRAMS } \\
\hline $\begin{array}{l}\text { Natural Resources Manageme } \\
\text { and Policy (USFSM) }\end{array}$ & 03.0201 & STEM & None & $0 \%$ & 65 & Spring 2020 \\
\hline $\begin{array}{l}\text { International Global Studies } \\
\text { (USFSM) }\end{array}$ & 30.2001 & GLOBAL & $\begin{array}{l}\text { NCF, UCF, UF, } \\
\text { UNF }\end{array}$ & $0 \%$ & 65 & Spring 2020 \\
\hline Design (USFT) & 50.0499 & NONE & None & $20 \%$ & 70 & Spring 2020 \\
\hline \multicolumn{7}{|c|}{ MASTER'S, SPECIALIST AND OTHER ADVANCED MASTER'S PROGRAMS } \\
\hline $\begin{array}{l}\text { Secondary Education and } \\
\text { Teaching (USFSP) }\end{array}$ & 13.1205 & EDUCATION & $\begin{array}{l}\text { FAU, FSU, } \\
\text { UCF, UNF }\end{array}$ & $80 \%$ & 20 & Spring 2020 \\
\hline $\begin{array}{l}\text { Marriage and Family } \\
\text { Therapy/Counseling (USFT) }\end{array}$ & 51.1505 & HEALTH & UCF, UF & $0 \%$ & 40 & Spring 2020 \\
\hline $\begin{array}{l}\text { Financial Planning and } \\
\text { Services (USFT) }\end{array}$ & 52.0804 & None & None & $0 \%$ & 40 & Spring 2020 \\
\hline Management Science (USFT) & 52.1301 & STEM & FSU & $0 \%$ & 100 & Spring 2020 \\
\hline \multicolumn{7}{|l|}{ DOCTORAL PROGRAMS } \\
\hline PhD Pharmacy (USF) & 51.2099 & EALTH & FAMU, UF & $0 \%$ & TBD & \\
\hline $\begin{array}{l}\text { OTD Occupational } \\
\text { Therapy/Therapist (USF) }\end{array}$ & 51.2306 & EALTH & UF & $0 \%$ & TBD & \\
\hline
\end{tabular}




\section{GLOSSARY}

\section{Performance Based Funding}

\section{Percent of Bachelor's Graduates Enrolled or Employed $(\$ 25,000+)$} One Year After Graduation
This metric is based on the percentage of a graduating class of bachelor's degree recipients who are enrolled or employed (earning at least $\$ 25,000$ ) somewhere in the United States. Students who do not have valid social security numbers and are not found enrolled are excluded. This data now includes non-Florida data from 41 states and districts, including the District of Columbia and Puerto Rico. Sources: State University Database System (SUDS), Florida Education \& Training Placement Information Program (FETPIP) and Florida Department of Economic Opportunity (DEO) analysis of Wage Record Interchange System (WRIS2) and Federal Employment Data Exchange (FEDES), and National Student Clearinghouse (NSC).

\section{Median Wages of Bachelor's Graduates Employed Full-time} One Year After Graduation
This metric is based on annualized Unemployment Insurance (UI) wage data from the fourth fiscal quarter after graduation for bachelor's recipients. This data does not include individuals who are self-employed, employed by the military, those without a valid social security number, or making less than minimum wage. This data now includes non-Florida data from 41 states and districts, including the District of Columbia and Puerto Rico. Sources: State University Database System (SUDS), Florida Education \& Training Placement Information Program (FETPIP) and Florida Department of Economic Opportunity (DEO) analysis of Wage Record Interchange System (WRIS2) and Federal Employment Data Exchange (FEDES), and National Student Clearinghouse (NSC).

\section{Cost to the Student} Net Tuition \& Fees for Resident Undergraduates per 120 Credit Hours
This metric is based on resident undergraduate student tuition and fees, books and supplies as calculated by the College Board (which serves as a proxy until a university work group makes an alternative recommendation), the average number of credit hours attempted by students who were admitted as FTIC and graduated with a bachelor's degree for programs that requires 120 credit hours, and financial aid (grants, scholarships and waivers) provided to resident undergraduate students (does not include unclassified students). Source: State University Database System (SUDS), the Legislature's annual General Appropriations Act, and university required fees.

\section{Four Year FTIC Graduation Rate}

This metric is based on the percentage of first-time-in-college (FTIC) students who started in the Fall (or summer continuing to Fall) term and were enrolled full-time in their first semester and had graduated from the same institution by the summer term of their fourth year. FTIC includes 'early admits' students who were admitted as a degree-seeking student prior to high school graduation. Source: State University Database System (SUDS).

\section{Academic Progress Rate} 2nd Year Retention with 2.0 GPA or Above
This metric is based on the percentage of first-time-in-college (FTIC) students who started in the Fall (or summer continuing to Fall) term and were enrolled full-time in their first semester and were still enrolled in the same institution during the Fall term following their first year with had a grade point average (GPA) of at least 2.0 at the end of their first year (Fall, Spring, Summer).

Source: State University Database System (SUDS). 


6. University Access Rate
Percent of Undergraduates
with a Pell-grant
7. Bachelor's Degrees within
Programs of Strategic
Emphasis

8a. Graduate Degrees within Programs of Strategic Emphasis 8b. Freshmen in Top 10\% of High School Class Applies only to: NCF
This metric is based the number of undergraduates, enrolled during the fall term, who received a Pell-grant during the fall term. Unclassified students, who are not eligible for Pell-grants, were excluded from this metric. Source: State University Database System (SUDS).

This metric is based on the number of baccalaureate degrees awarded within the programs designated by the Board of Governors as 'Programs of Strategic Emphasis'. A student who has multiple majors in the subset of targeted Classification of Instruction Program codes will be counted twice (i.e., double-majors are included).

Source: State University Database System (SUDS).

This metric is based on the number of graduate degrees awarded within the programs designated by the Board of Governors as 'Programs of Strategic Emphasis'. A student who has multiple majors in the subset of targeted Classification of Instruction Program codes will be counted twice (i.e., double-majors are included).

Source: State University Database System (SUDS).

Percent of all degree-seeking, first-time, first-year (freshman) students who had high school class rank within the top $10 \%$ of their graduating high school class.

Source: New College of Florida as reported to the Common Data Set.

\section{BOG Choice Metric}

\section{Percent of Bachelor's Degrees Without Excess Hours}

This metric is based on the percentage of baccalaureate degrees awarded within $110 \%$ of the credit hours required for a degree based on the Board of Governors Academic Program Inventory. Note: It is important to note that the statutory provisions of the "Excess Hour Surcharge" (1009.286, FS) have been modified several times by the Florida Legislature, resulting in a phased-in approach that has created three different cohorts of students with different requirements. The performance funding metric data is based on the latest statutory requirements that mandates $110 \%$ of required hours as the threshold. In accordance with statute, this metric excludes the following types of student credits (ie, accelerated mechanisms, remedial coursework, non-native credit hours that are not used toward the degree, non-native credit hours from failed, incomplete, withdrawn, or repeated courses, credit hours from internship programs, credit hours up to 10 foreign language credit hours, and credit hours earned in military science courses that are part of the Reserve Officers' Training Corps (ROTC) program). Source: State University Database System (SUDS).

\section{BOT Choice Metrics}

10a. Percent of R\&D Expenditures Funded from External Sources FAMU

10b. Bachelor's Degrees Awarded to Minorities FAU, FGCU, FIU
This metric reports the amount of research expenditures that was funded from federal, private industry and other (non-state and non-institutional) sources.

Source: National Science Foundation annual survey of Higher Education Research and Development (HERD).

This metric is the number, or percentage, of baccalaureate degrees granted in an academic year to Non-Hispanic Black and Hispanic students. This metric does not include students classified as Non-Resident Alien or students with a missing race code. Source: State University Database System (SUDS). 


\section{0c. National Rank Higher than Predicted by the Financial Resources Ranking Based on U.S. and World News FSU}

This metric is based on the difference between the Financial Resources rank and the overall University rank. U.S. News measures financial resources by using a two-year average spending per student on instruction, research, student services and related educational expenditures - spending on sports, dorms and hospitals doesn't count. Source: US News and World Report's annual National University rankings.

\section{0d. Percent of Undergraduate Seniors Participating in a Research Course NCF}

\section{0e. Number of Bachelor Degrees Awarded Annually UCF}

This metric is based on the percentage of undergraduate seniors who participate in a research course during their senior year.

Source: New College of Florida.
This metric is the number of baccalaureate degrees granted in an academic year. Students who earned two distinct degrees in the same academic year were counted twice; students who completed multiple majors or tracks were only counted once.

Source: State University Database System (SUDS).

\section{0f. Number of Licenses/Options Executed Annually UF} This metric is the total number of licenses and options executed annually as reported to
Association of Technology Managers (AUTM). The benchmarks are based on UF's national
rank among public \& private institutions. Source: University of Florida.

\section{0g. Percent of Undergraduate FTE in Online Courses UNF}

This metric is based on the percentage of undergraduate full-time equivalent (FTE) students enrolled in online courses. The FTE student is a measure of instructional activity that is based on the number of credit hours that students enroll by course level. Distance Learning is a course in which at least 80 percent of the direct instruction of the course is delivered using some form of technology when the student and instructor are separated by time or space, or both (per 1009.24(17), F.S.).

Source: State University Database System (SUDS).

\section{Number of Postdoctoral Appointees USF}

This metric is based on the number of post-doctoral appointees during the Fall term of the academic year. A postdoctoral researcher has recently earned a doctoral (or foreign equivalent) degree and has a temporary paid appointment to focus on specialized research/scholarship under the supervision of a senior scholar.

Source: National Science Foundation/National Institutes of Health annual Survey of Graduate Students and Post-doctorates in Science and Engineering (GSS).

\section{Percentage of Adult Undergraduates Enrolled UWF}

This metric is based on the percentage of undergraduates (enrolled during the fall term) who are at least 25 years old at the time of enrollment. This includes undergraduates who are not degree-seeking, or unclassified.

Source: State University Database System (SUDS).

\section{Preeminent Research University Funding Metrics}

\author{
Average GPA and SAT Score
}

An average weighted grade point average of 4.0 or higher and an average SAT score of 1200 or higher for fall semester incoming freshmen, as reported annually in the admissions data that universities submit to the Board of Governors. This data includes registered FTIC (student type='B','E') with an admission action of admitted or provisionally admitted ('A','P','X'). Source: State University Database System (SUDS). 
Public University National
Ranking

Freshman Retention Rate

(Full-time, FTIC)

6-year Graduation Rate

(Full-time, FTIC)

A top-50 ranking on at least two well-known and highly respected national public university rankings, reflecting national preeminence, using most recent rankings, includes: Princeton Review, Fiske Guide, QS World University Ranking, Times Higher Education World University Ranking, Academic Ranking of World University, US News and World Report National University, US News and World Report National Public University, US News and World Report Liberal Arts Colleges, Forbes, Kiplinger, Washington Monthly Liberal Arts Colleges, Washington Monthly National University, and Center for Measuring University Performance.

Freshman Retention Rate (Full-time, FTIC) as reported annually to the Integrated Postsecondary Education Data System (IPEDS).

Cohorts are based on undergraduate students who enter the institution in the Fall term (or Summer term and continue into the Fall term). Percent Graduated is based on federal rate and does not include students who originally enroll as part-time students, or who transfer into the institution.

National Academy Memberships held by faculty as reported by the Center for Measuring University Performance in the Top American Research Universities (TARU) annual report or the official membership directories maintained by each national academy.

Science \& Engineering Research Expenditures (\$M)

Science \& Engineering Research Expenditures, including federal research expenditures as reported annually to the National Science Foundation (NSF).

\section{Non-Medical Science \& Engineering Research Expenditures (\$M)}

National Ranking in S.T.E.M. Research Expenditures

Patents Awarded

(3 calendar years)

\section{Doctoral Degrees} Awarded Annually

Number of Post-Doctoral Appointees
Total S\&E research expenditures in non-medical sciences as reported to the National Science Foundation (NSF). This removes medical sciences funds from the total S\&E amount.

The NSF identifies 8 broad disciplines within Science \& Engineering (Computer Science, Engineering, Environmental Science, Life Science, Mathematical Sciences, Physical Sciences, Psychology, Social Sciences). The rankings by discipline are determined by BOG staff using the NSF WebCaspar database.

Total utility patents awarded by the United States Patent and Trademark Office (USPTO) for the most recent three calendar year period. Due to a year-lag in published reports, Board of Governors staff query the USPTO database with a query that only counts utility patents:"(AN/"University Name" AND ISD/yyyymmdd->yyyymmdd AND APT/1)".

Doctoral research degrees awarded annually as reported annually by the Board of Governors. The Legislature excluded professional doctoral degrees from this metric. The 2016 Legislature amended this criteria to include professional doctoral degrees awarded in medical and health care disciplines.

The number of Postdoctoral Appointees awarded annually, as reported in the TARU annual report. This data is based on National Science Foundation/National Institutes of Health annual Survey of Graduate Students and Post-doctorates in Science and Engineering (GSS). 
This data comes from the National Association of College and University Business Officers (NACUBO) and Commonfund Institute's annual report of Market Value of Endowment Assets.

\section{Key Performance Indicators}

\section{Teaching \& Learning Metrics}

Freshmen in Top 10\% of HS Graduating Class

\section{Professional/Licensure Exam First-time Pass Rates}

\section{Average Time to Degree} for FTIC in $120 \mathrm{hr}$ programs

\section{Six-Year Graduation Rates}

Percent of all degree-seeking, first-time, first-year (freshman) students who had high school class rank within the top $10 \%$ of their graduating high school class. Source: As reported by the university to the Common Data Set.

The average pass rates as a percentage of all first-time examinees for Nursing, Law, Medicine (3 subtests), Veterinary, Pharmacy, Dental ( 2 subtests), Physical Therapy, and Occupational Therapy, when applicable. The average pass rate for the nation or state is also provided as a contextual benchmark. The Board's 2025 System Strategic Plan calls for all institutions to be above or tied the exam's respective benchmark. Note about Benchmarks: The State benchmark for the Florida Bar Exam excludes non-Florida institutions. The national benchmark for the USMLE exams are based on rates for MD degrees from US institutions.

This metric is the number of years between the start date (using the student entry date) and the end date (using the last month in the term degree was granted) for a graduating class of first-time, single-major baccalaureates in 120 credit hour programs within a (Summer, Fall, Spring) year. Source: State University Database System (SUDS).

The First-time-in-college (FTIC) cohort is defined as undergraduates entering in fall term (or summer continuing to fall) with fewer than 12 hours earned since high school graduation. The rate is the percentage of the initial cohort that has either graduated from the same institution by the summer term of their sixth academic year. Both fulltime and part-time students are used in the calculation. FTIC includes 'early admits' students who were admitted as a degree-seeking student prior to high school graduation. Source: State University Database System (SUDS).

This is a count of first-major baccalaureate and graduate degrees awarded. First Majors include the most common scenario of one student earning one degree in one Classification of Instructional Programs (CIP) code. In those cases where a student earns a baccalaureate degree under two different degree CIPs, a distinction is made between "dual degrees" and "dual majors." Also included in first majors are "dual degrees" which are counted as separate degrees (e.g., counted twice). In these cases, both degree CIPs receive a "degree fraction" of 1.0. The calculation of degree fractions is made according to each institution's criteria. Source: State University Database System (SUDS).

Race/Ethnicity data is self-reported by students. Non-Hispanic Black and Hispanic do not include students classified as Non-Resident Alien or students with a missing race code. Degree data is based on first-major counts only - second majors are not included. Percentage of Degrees is based on the number of baccalaureate degrees awarded to non-Hispanic Black and Hispanic students divided by the total degrees awarded excluding those awarded to non-resident aliens and unreported. Source: State University Database System (SUDS). 


\section{Adult (Aged 25+) Undergraduates Enrolled Fall term}

This metric is based on the age of the student at the time of their Fall term enrollment - not their age upon entry. As a proxy, age is based on birth year not birth date. Note: Unclassified students with a HS diploma (or GED) and above are included in this calculation. Source: State University Database System (SUDS).
Percent of Undergraduate FTE Enrolled in Online Courses

Full-time Equivalent (FTE) student is a measure of instructional activity that is based on the number of credit hours that students enroll. FTE is based on the US definition, which divides undergraduate credit hours by 30 . Distance Learning is a course in which at least 80 percent of the direct instruction of the course is delivered using some form of technology when the student and instructor are separated by time or space, or both (per 1009.24(17), F.S.). Source: State University Database System (SUDS).

The percentage of baccalaureate degrees that are classified as STEM or Health disciplines by the Board of Governors in the Academic Program Inventory. These counts include second majors. Second Majors include all dual/second majors (e.g., degree CIP receive a degree

Percent of Bachelor's And Graduate Degrees in STEM \& Health fraction that is less than 1). The calculation of degree fractions is made according to each institution's criteria. The calculation for the number of second majors rounds each degree CIP's fraction of a degree up to 1 and then sums the total. Second Majors are typically used when providing degree information by discipline/CIP, to better conveys the number of graduates who have specific skill sets associated with each discipline. Source: State University Database System (SUDS).

\section{Scholarship, Research \& Innovation Metrics}

National Academy
Members

Faculty Awards

Total Research Expenditures (\$M)

Percent of R\&D Expenditures funded from External Sources

Utility Patents Awarded

Licenses/Options Executed

Number of Start-up Companies
National Academy Memberships held by faculty as reported by the Center for Measuring University Performance in the Top American Research Universities (TARU) annual report or the official membership directories maintained by each national academy.

Awards include: American Council of Learned Societies (ACLS) Fellows, Beckman Young Investigators, Burroughs Wellcome Fund Career Awards, Cottrell Scholars, Fulbright American Scholars, Getty Scholars in Residence, Guggenheim Fellows, Howard Hughes Medical Institute Investigators, Lasker Medical Research Awards, MacArthur Foundation Fellows, Andrew W. Mellon Foundation Distinguished Achievement Awards, National Endowment for the Humanities (NEH) Fellows, National Humanities Center Fellows, National Institutes of Health (NIH) MERIT, National Medal of Science and National Medal of Technology, NSF CAREER awards (excluding those who are also PECASE winners), Newberry Library Long-term Fellows, Pew Scholars in Biomedicine, Presidential Early Career Awards for Scientists and Engineers (PECASE), Robert Wood Johnson Policy Fellows, Searle Scholars, Sloan Research Fellows, Woodrow Wilson Fellows.

Total expenditures for all research activities (including non-science and engineering activities) as reported in the National Science Foundation annual survey of Higher Education Research and Development (HERD).

This metric reports the amount of research expenditures that was funded from federal, private industry and other (non-state and non-institutional) sources.

Source: National Science Foundation annual survey of Higher Education Research and Development (HERD).

The number of utility patents awarded by the United States Patent and Trademark Office (USPTO) by Calendar year - does not include design, plant or other types.

Licenses/options executed in the fiscal year for all technologies - as reported by universities on the Association of University Technology Managers Annual (AUTM) annual Licensing Survey.

The number of start-up companies that were dependent upon the licensing of University technology for initiation. 